\title{
Effect of Coating and Wrapping Materials on Storage Behavior of
} Balady Mandarin Fruits

Fatma El-Zahraa M. Gouda ${ }^{1}$ and Sabah M. Badawy ${ }^{2}$

${ }^{1}$ Pomology Department, Fac. Agric, Assiut University

${ }^{2}$ Agricultural Research Center, Shandaweel, Sohag

Received on: $29 / 6 / 2016$

Accepted for publication on: 29/7/2016

\section{Abstract}

This study was conducted during two successive seasons 2014 \& 2015 to investigate the effect of coating and wrapping materials on storability and quality of Balady mandarin fruits during the storage period. Fruits were coated with salicylic acid, paraffin oil or camphor oil, as well as wrapped with foil paper and untreated. The fruits were stored either at room temperature or cold conditions $\left(0 \pm 2^{\circ} \mathrm{C}\right)+$ humidity of $90 \pm 5 \%$.

The results showed that the fruit weight loss $\%$, fruit decay $\%$, total soluble solids $\%$ and TSS/acid ratio were significantly increased with prolonging the storage period. On the other hand, the advanced storage period induced a gradual decrease of fruit juice percentage acidity $\%$, vitamin $\mathrm{C}$ and total phenolic contents levels.

All treatments caused decreased fruit weight loss and fruit decay percentage under both storage conditions. Using foil paper wrapping or salicylic acid gave the least fruit weight loss and decayed fruit percentage, respectively. The most loss reduction was associated with the cold storage than room temperature conditions. Also, all treatments improved the fruit quality during the storage period compared to the untreated ones.

So, it could be concluded that oil coating or wrapping maintained fruit freshness without negative effects on fruit quality parameters. Meanwhile, coating and wrapping fruits retained high levels of juice volume, acidity, ascorbic acid (V. C) and total phenolic contents as well as lower total soluble solids and TSS/acid ratio loss than controls during storage. Such treatments can be used as alternative of chemical application in order to maintain human health and environment.

Keyword: Coating, Wrapping, Salicylic acid, Storability, Fruit quality storage, Balady mandarin.

\section{Introduction}

Citrus fruits are important part of human diet because of their nutritional value, antioxidant activity and high consumption. Citrus fruits are rich in vitamin $\mathrm{C}$ and also contain large amounts of phytochemical (Heber and $\mathrm{Lu}, 2002$ ). It is one of the most important fruit crops in the world. It ranks the third position between fruit crops and only preceded with grapes and apples. However, in
Egypt, citrus species are the most important fruit crop either for local consumption or exportation. Balady mandarin is one of the most important citrus species that Egyptian consumers and export market prefer because of its flavor and easy peeling. In developing countries, the postharvest decay destroys more than $30 \%$ of the crop yield. Storage temperature is the most important environmental factor affecting senescence 
of fruits, because it regulates the rate of physiological and bio-chemical processes (Kader, 2002).

During post-harvest storage, due to the internal and external factors, chemical and physical changes occur in fruits and vegetables, which results in losses in nutritional quality. To prevent these adverse effects, use of environmentally friendly technologies such as edible coating or salicylic acid are of prime important (Saucedo et al., 2007 Ladaniya, 2011). Salicylic acid exhibits a high potential in enhancing quality, controlling post-harvest losses and mitigating chilling injury in fruits and vegetables. It is known that, the cold storage plays an important role in prolonging shelf life of many fruits and vegetables with keeping their quality during storage. Economically, it is necessary to discover a cheap and effective storage method to increase its shelf-life by decreasing the natural physiological deterioration and preventing the activity of decay organisms (Aghdam and Bodbodak, 2013).

The application of surface coating on fruits is considered as one of several treatments developed to reduce post-harvest losses and to prolong self life of fruits. Surface coating has been used as preservation technique for fruits and vegetables (Baldwin et al., 1995). Surface coating of fruits reduce the water evaporation from the fruits and thereby slow the weight loss, can maintain the freshness of fruits and improve the appearance of them. Hence, surface coating on fruit, can maintain the quality of fruits and reduce the post harvest decay (Park, 1999).
Two major problems that limit facing the long term storage capability of citrus fruits are pathological and physiological breakdown leading to decay and weight loss (Kader, 2002). To solve the problem of decay development and weight loss, this needs either fungicide application or alternative environmental safe methods. The deterioration of citrus fruit during storage results mainly from transpiration and respiration (Baubaker et al., 2009).

Many plants extracts and essential oils were shown to have high antifungal activity against a wide range of fungi. Also, essential oils and botanical extract are commercially available as pre-harvest and postharvest fungicides for organic farming (Plooy et al., 2009 and Mansour, 2015). Individual oil film wrapping of fresh fruits will greatly reduce weight loss by reducing the transpiration and maintaining fruit firmness (Rodov et al., 2001; Abd El-Motty and El-Faham, 2013 and Mansour, 2015).

Therefore, this study aimed to recognize the benefit of using oil and salicylic acid coating and foil paper wrapping on storability of Balady mandarin fruits.

\section{Materials and Methods}

This study was conducted during two successive seasons of 2014 and 2015 on Balady mandarin (Citrus reticulata L.) fruits. The fruits were obtained from the Experimental Orchard of the Faculty of Agriculture, Assiut University.

Fruits were harvested in the first week of January, hand packed and carefully brought, soon after picking to laboratory of Pomology Depart- 
ment, Faculty of Agriculture, Assiut University. Fruits were selected for uniformity in maturity, shape, size and weight. The fruits were free from obvious defects or mechanical damage. All fruits were washed with regular tap water and soap and then rinsed with water to remove the residue of soap, then air dried. Fruits were divided into five similar groups and subjected to one of the following treatments.

1- Control group (untreated).

2- Fruits were coated with thin layer of salicylic acid at $0.4 \%$.

3- Fruits were coated with thin layer of paraffin oil at $20 \%$.

4- Fruits were coated with thin layer of camphor oil at $10 \%$.

5- Fruits were wrapped in foil paper $(0.05 \mathrm{~cm}$ thickness $)$.

Each treatment was replicated three times and each replicate put as one layer in a carton box. The half of experimental boxes were stored at $\left(5 \pm 2^{\circ} \mathrm{C}\right)$ and $(90 \% \pm 5)$ relative humidity and the other, at room temperature. Changes in some physical and chemical fruits properties were determined every 7 days at room temperature and every 15 days at cold storage temperature, respectively.

\section{Physical characteristics:}

1- Weight loss as a percentage from the initial weight.

2- Decay percentage: the number of decayed fruits was calculated as a percentage from the total number of each sample.

3- Juice percentage: juice content was expressed as percent of fruit weight (W/W).

\section{Chemical juice:}

1- The total soluble solids (TSS) were determined by using the hand refractometer.

2- Titratable acidity (TA) expressed as percentage of citric acid, by using $0.1 \mathrm{~N}$ sodium hydroxide according to the A.O.A.C. (1985) lated

3- TSS/TA ratio was then calcu-

4- L-ascorbic acid (V.C.) was determined by titration against 2,6dichlorophenol indophenol blue dye, according to the A.O.A.C. (1985).

5- Total phenolic were determined by Folin-Ciocalteu method (Singleton et al., 1999).

This experiment was conducted in complete randomized design with three replicate and with factorial arrangement was followed throughout the whole work. All recorded data were tabulated and statistically analyses according to Snedecor and Cochran (1990) using L.S.D. at the level of 0.05 in comparison between various treatments means.

\section{Results}

1- The percentage of fruit weight loss and decay fruits:

Data presented in Tables ( 1 \& 2 ) showed the effect of oil and salicylic acid coating and foil paper wrapping on the percentage of weight loss and decay of Balady mandarin fruits during the storage in 2014 and 2015 seasons. It was obvious that results took similar trend during the two studied seasons.

Data in the previous tables cleared that fruit weight loss, as well as, decayed fruits were markedly increased with the advancement of room temperature and cold storage period. These traits were slightly increased gradually from the beginning 
either in room temperature or cold till the week five or the third month, respectively.

In response to treatments, it was apparent that all treatments significantly reduced the fruit weight loss percentage and undesirable fruit decay percentage under both storage conditions compared with controls during the two studied seasons. Using foil paper wrapping had the best results, which gave the least percentage of fruit weight loss (9.14 or $9.63 \%)$ and $(5.63 \& 5.73 \%)$ against $(12.68 \&$ $12.83 \%)$ and (10.63 \& 10.95\%) on untreated ones of storage under room temperature or cold during the two studied seasons, respectively. The decrement of percentage of weight loss due to foil paper wrapping compared to controls attained $(27.92 \&$ 24.94) and (48.01 \& 47.67\%) under room temperature and cold storage during the two studied seasons, respectively. On the other hand, using salicylic acid gave the least decayed fruits percentage $(27.77 \& 28.88 \%)$ and $(21.94 \& 23.33 \%)$ versus $(52.22$ $\& 54.44 \%$ ) and (48.33 \& 50.67\%) under room temperature and cold storage during the two studied seasons, respectively. The corresponding decrement percentage attained (46.82 $\& 46.95 \%)$ and (54.60 \& 53.96\%) during the two studied seasons, respectively.

Moreover, storage under cold condition had the least percentages of fruit weight loss and decayed fruits than storage under room temperature. Also, the variation in reduction of fruit weight loss and decayed fruits depends on the natural coating. Foil paper wrapping gave the least percentage of fruit weight loss whereas, the salicylic acid coating gave the least percentage of decayed fruits compared to the other treatments under both storage conditions.

Table 1. Effect of coating and wrapping materials on weight loss of Balady mandarin fruits during storage in 2014 and 2015 seasons.

\begin{tabular}{|c|c|c|c|c|c|c|c|c|c|c|c|c|c|c|c|}
\hline \multirow{3}{*}{$\begin{array}{r}\begin{array}{r}\text { Period } \\
\text { (A) }\end{array} \\
\text { Treat. (B) }\end{array}$} & \multicolumn{7}{|c|}{ Room temperature } & \multicolumn{8}{|c|}{ Cold storages } \\
\hline & \multicolumn{7}{|c|}{ Weekly } & \multicolumn{8}{|c|}{ Biweekly } \\
\hline & $\mathbf{P 0}$ & 1 & 2 & 3 & 4 & 5 & Mean & P0 & 1 & 2 & 3 & 4 & 5 & 6 & Mean \\
\hline \multicolumn{16}{|c|}{2014} \\
\hline alic & 0.0 & 1.91 & 5. & & 7.55 & 10.20 & 5.14 & 0.0 & 0.68 & 2.18 & 2.90 & 4.35 & 4.75 & .88 & \\
\hline ara & & 1.80 & 4. & & 7.21 & & & 0.0 & 0.90 & 66 & 3.65 & 4.66 & 6.15 & & \\
\hline & .0 & 2.10 & 4.45 & 01 & 7.53 & & & 0.0 & 0.72 & 33 & 3.18 & 4.67 & 5.81 & & \\
\hline oil p & 0.0 & 1.50 & 4.36 & 5.18 & 6.57 & 9.14 & 4.46 & 0.0 & 0.93 & 2.50 & 3.21 & 4.31 & 5.10 & 3 & \\
\hline n & 0.0 & 2.39 & 6.77 & 7.89 & 9.46 & 12.68 & 6.53 & 0.0 & 1.32 & 3.76 & 5.26 & 6.35 & 8.35 & 10.83 & 5.12 \\
\hline 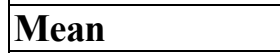 & 0.0 & 1.94 & 5.12 & 6.22 & 7.66 & 10.46 & & 0.0 & 0.91 & 2.69 & 3.64 & 4.87 & 6.03 & 7.97 & \\
\hline & A: & 0.16 & B: & 0.19 & AB: & 0.46 & & A: & 0.11 & B: & 0.13 & AB: & & & \\
\hline \multicolumn{16}{|c|}{2015} \\
\hline & & 65 & & & 7.95 & & 5.32 & & 0.82 & 2.51 & 3.65 & 5.23 & 5.86 & 7.10 & \\
\hline & 0.0 & 1.52 & 4.88 & 6.05 & 7.40 & & 5.01 & 0.0 & 1.20 & 2.80 & 4.18 & 5.25 & 7.10 & & \\
\hline & 0.0 & 1.89 & 4.62 & 6.28 & 7.79 & 10.48 & 5.18 & 0.0 & 0.90 & 2.86 & 3.80 & 5.75 & 6.48 & 1 & \\
\hline oil paper & 0.0 & 2.18 & 4.02 & \begin{tabular}{|l|}
5.10 \\
\end{tabular} & 6.34 & 9.63 & 4,55 & 0.0 & \begin{tabular}{|l|}
1.16 \\
\end{tabular} & 2.72 & 3.62 & 4.81 & 5.18 & 5.73 & 3.32 \\
\hline ontrol & 0.0 & 2.56 & 6.28 & 8.22 & 9.65 & 12.83 & 6.59 & 0.0 & \begin{tabular}{|l|}
1.50 \\
\end{tabular} & 4.16 & \begin{tabular}{|l|}
5.62 \\
\end{tabular} & 6.85 & 7.50 & 10.95 & 5.23 \\
\hline Mean & 0.0 & 1.96 & 5.01 & 6.39 & 7.83 & 10.76 & & 0.0 & 1.12 & 3.01 & 4.19 & 5.58 & 6.42 & 7.95 & \\
\hline 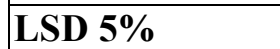 & A: & 0.18 & B: & 0.22 & AB: & & & A: & 0.13 & B: & 0.15 & AB: & & 0.37 & \\
\hline
\end{tabular}

$\mathrm{A}=$ Period.

B: Treat 
Table 2. Effect of coating and wrapping materials on decay \% of Balady mandarin fruits during storage in 2014 and 2015 seasons.

\begin{tabular}{|c|c|c|c|c|c|c|c|c|c|c|c|c|c|c|c|}
\hline \multirow{3}{*}{ Treat. (B) } & \multicolumn{7}{|c|}{ Room temperature } & \multicolumn{8}{|c|}{ Cold storages } \\
\hline & \multicolumn{7}{|c|}{ Weekly } & \multicolumn{8}{|c|}{ Biweekly } \\
\hline & P0 & 1 & 2 & 3 & 4 & 5 & Mean & P0 & 1 & 2 & 3 & 4 & 5 & 6 & Mean \\
\hline \multicolumn{16}{|c|}{2014} \\
\hline Salicylic acid & 0.0 & 0.0 & 0.0 & 1.22 & 6.67 & 27.77 & 5.94 & 0.0 & 0.0 & 1.22 & 8.89 & 15.55 & 17.78 & 21.94 & 9.34 \\
\hline Paraffin oil & 0.0 & 0.0 & 0.0 & 3.33 & 10.0 & 33.74 & 9.84 & 0.0 & 0.0 & 3.33 & 11.11 & 20.10 & 21.11 & 27.18 & 11.82 \\
\hline Camphor oil & 0.0 & 0.0 & 0.0 & 2.22 & 6.67 & 28.88 & 6.29 & 0.0 & 0.0 & 2.44 & 8.89 & 16.67 & 16.67 & 22.22 & 9.56 \\
\hline Foil paper & 0.0 & 0.0 & 2.22 & 8.88 & 13.33 & 38.88 & 10.55 & 0.0 & 0.0 & 5.55 & 12.22 & 16.67 & 24.44 & 32.45 & 13.05 \\
\hline Control & 0.0 & 3.33 & 10.0 & 15.55 & 20.00 & 52.22 & 16.85 & 0.0 & 3.33 & 9.76 & 15.56 & 27.78 & 42.22 & 48.33 & 21.00 \\
\hline Mean & 0.0 & 0.67 & 2.44 & 6.24 & 11.33 & 36.30 & & 0.0 & 0.67 & 4.46 & 11.33 & 19.33 & 24.44 & 39.42 & \\
\hline LSD 5\% & A: & 0.81 & B: & 0.98 & AB: & 2.41 & & A: & 1.28 & B: & 1.51 & AB: & 3.68 & & \\
\hline \multicolumn{16}{|c|}{2015} \\
\hline Salicylic acid & 0.0 & 0.0 & 0.0 & 2.44 & 7.77 & 28.88 & 6.52 & 0.0 & 0.0 & 1.44 & 8.88 & 16.67 & 18.89 & 23.33 & 11.53 \\
\hline Paraffin oil & 0.0 & 0.0 & 0.0 & 4.44 & 11.11 & 35.55 & 8.52 & 0.0 & 0.0 & 3.33 & 13.33 & 20.00 & 22.22 & 28.89 & 14.63 \\
\hline Camphor oil & 0.0 & 0.0 & 0.0 & 3.33 & 8.88 & 3.09 & 7.05 & 0.0 & 0.0 & 2.44 & 10.0 & 16.67 & 17.78 & 23.33 & 11.80 \\
\hline Foil paper & 0.0 & 0.0 & 1.22 & 8.88 & 13.33 & 40.00 & 10.57 & 0.0 & 0.0 & 6.67 & 11.11 & 16.67 & 25.55 & 34.44 & 15.74 \\
\hline Control & 0.0 & 4.44 & 11.11 & 17.77 & 22.22 & 54.44 & 18.33 & 0.0 & 3.33 & 10.00 & 16.67 & 26.67 & 43.33 & 50.67 & 25.25 \\
\hline Mean & 0.0 & 0.89 & 2.47 & 7.37 & 12.66 & 37.79 & & 0.0 & 0.67 & 4.78 & 11.99 & 19.34 & 25.55 & 32.13 & \\
\hline LSD 5\% & A: & 0.88 & B: & 1.05 & AB: & 2.53 & & A: & 0.99 & B: & 1.16 & AB: & 2.61 & & \\
\hline
\end{tabular}

The results indicated that the fruit wrapping or coating in combination with low temperature storage proved effective in reducing the percentage of weight loss and decayed fruits as well as keeping the Balady mandarin fruits for longest period.

2- The percentage juice contents:

The data introduced in Table (3) disclosed that fruit juice percentage was significantly decreased by extending cooling storage duration. The highest values were recorded at the beginning of storage, whereas, the least ones was recorded at the end of storage course under both storage conditions.

Storage under cold condition had the least reduction of juice percentage compared to storage under room temperature.

All treatments lead to significantly increased fruit juice percentage compared to controls under both storage conditions. No significant differences were found between treatments in the different storage conditions during both the studied seasons. Foil paper wrapping gave the best effect of preserving the juice percentage (33.35 \& 33.53\%) under room temperature storage as well as $(33.15 \&$ $33.20 \%$ ) under cold storage during the two studied seasons, respectively. All treatments were retained the juice volume higher as possible during storage compared with controls which gave the lowest juice percentage $(30.20 \& 30.80 \%)$ and $(30.65 \&$ $30.45 \%$ ) under room temperature and cold storage conditions during two studied seasons, respectively.

\section{3- Fruit chemical properties:}

The data concerning the effect of oil, salicylic acid coating and foil paper wrapping on the chemical constituents of Balady mandarin juice during the storage condition in 2014 and 2015 seasons are presented in Tables (4 to 8 ). It was obvious from the data that results took similar trends during the three studied seasons.

In a general view, it could be noticed that TSS and TSS/acid ratio were gradually increased in all the 
stored fruits with the extending storage period until five weeks at room temperature and three months under cold storage. On the other hand, prolonging the storage for five weeks or three months induce a gradually decrease of the titratable acidity, ascorbic acid (V.C) and total phenolic contents.

According to the treatment effects, it is clear from the previous data that, all treatments lead to sig- nificant effects on chemical juice properties compared to untreated fruits under both storage conditions. Moreover, no significant differences were found in chemical juice quality among all treatments. At the end of storage periods, there is a difference in TSS and TSS/acid occurred between the two storage temperatures with higher values under room temperature than that under the cold storage.

Table 3. Effect of coating and wrapping materials on juice weight $\%$ of Balady mandarin fruits during storage in 2014 and 2015 seasons.

\begin{tabular}{|c|c|c|c|c|c|c|c|c|c|c|c|c|c|c|c|}
\hline \multirow{3}{*}{ Treat. (B) } & \multicolumn{7}{|c|}{ Room temperature } & \multicolumn{8}{|c|}{ Cold storages } \\
\hline & \multicolumn{7}{|c|}{ Weekly } & \multicolumn{8}{|c|}{ Biweekly } \\
\hline & P0 & 1 & 2 & 3 & 4 & 5 & Mean & P0 & 1 & 2 & 3 & 4 & 5 & 6 & Mean \\
\hline \multicolumn{16}{|c|}{2014} \\
\hline Salicylic acid & 37.80 & 36.85 & 36.25 & 35.71 & 34.90 & 31.96 & 35.58 & 37.80 & 36.30 & 36.10 & 35.45 & 34.60 & 32.98 & 31.60 & 34.37 \\
\hline Paraffin oil & 37.80 & 37.15 & 36.62 & 36.10 & 35.28 & 32.18 & 35.85 & 37.80 & 36.70 & 36.25 & 35.81 & 34.85 & 33.17 & 31.75 & 35.19 \\
\hline Camphor oil & 37.80 & 36.90 & 36.16 & 35.61 & 34.68 & 31.85 & 35.40 & 37.80 & 36.87 & 36.13 & 35.33 & 35.10 & 33.28 & 31.53 & 35.15 \\
\hline Foil paper & 37.80 & 37.10 & 36.62 & 35.85 & 35.10 & 33.35 & 35.97 & 37.80 & 36.90 & 36.33 & 35.15 & 35.88 & 33.80 & 33.15 & 35.56 \\
\hline Control & 37.80 & 35.80 & 34.75 & 33.95 & 33.40 & 30.20 & 34.32 & 37.80 & 36.15 & 35.24 & 34.38 & 32.80 & 31.85 & 30.65 & 34.12 \\
\hline Mean & 37.80 & 36.76 & 36.08 & 35.45 & 34.66 & 31.91 & & 37.80 & 36.58 & 36.01 & 35.22 & 34.65 & 33.02 & 31.74 & \\
\hline LSD 5\% & \multicolumn{2}{|c|}{ A: 1.28} & B: 1.0 & 1.09 & \multicolumn{2}{|c|}{\begin{tabular}{|l|l|} 
AB: & 2.67 \\
\end{tabular}} & & A: 11 & \begin{tabular}{l|l}
.52 & \\
\end{tabular} & B: 1. & 33 & AB: & 3.26 & & \\
\hline \multicolumn{16}{|c|}{2015} \\
\hline Salicylic acid & 36.18 & 35.30 & 34.96 & 34.13 & 33.90 & 32.48 & 34.49 & 36.18 & 39.35 & 34.64 & 34.10 & 33.25 & 32.60 & 31.89 & 33.97 \\
\hline Paraffin oil & 36.18 & 35.80 & 35.28 & 34.65 & 34.18 & 32.70 & 34.80 & 36.18 & 35.60 & 34.80 & 34.45 & 33.60 & 32.85 & 32.10 & 34.23 \\
\hline Camphor oil & 36.18 & 34.53 & 35.10 & 34.10 & 33.30 & 32.20 & 34.34 & 36.18 & 35.52 & 34.60 & 34.00 & 33.18 & 33.10 & 32.37 & 34.18 \\
\hline Foil paper & 36.18 & 35.45 & 35.98 & 34.35 & 34.28 & 33.53 & 34.96 & 36.18 & 34.90 & 35.10 & 34.50 & 34.25 & 33.50 & 33.20 & 34.66 \\
\hline Control & 36.18 & 34.65 & 33.60 & 32.84 & 32.55 & 30.80 & 33.44 & 36.18 & 35.18 & 34.28 & 34.19 & 32.11 & 31.68 & 30.15 & 33.25 \\
\hline Mean & 36.18 & 36.16 & 34.58 & 34.01 & 33.68 & 32.30 & & 36.18 & 35.51 & 34.68 & 34.05 & 33.28 & 32.75 & 32.00 & \\
\hline LSD 5\% & \multicolumn{2}{|l|}{\begin{tabular}{l|l} 
A: & 1 \\
\end{tabular}} & B: 0.9 & 0.98 & \multicolumn{2}{|c|}{\begin{tabular}{l|l|}
$\mathbf{A B}:$ & 2.37 \\
\end{tabular}} & & A: & 1.41 & B: 1 . & 1.15 & AB: & \multicolumn{2}{|c|}{2.83} & \\
\hline
\end{tabular}


Table 4. Effect of coating and wrapping materials on total soluble solids of Balady mandarin fruits during storage in 2014 and 2015 seasons.

\begin{tabular}{|c|c|c|c|c|c|c|c|c|c|c|c|c|c|c|c|}
\hline \multirow{3}{*}{$\begin{array}{r}\text { Period } \\
\text { Treat. (B) }\end{array}$} & \multicolumn{7}{|c|}{ Room temperature } & \multicolumn{8}{|c|}{ Cold storages } \\
\hline & \multicolumn{7}{|c|}{ Weekly } & \multicolumn{8}{|c|}{ Biweekly } \\
\hline & P0 & 1 & 2 & 3 & 4 & 5 & Mean & P0 & 1 & 2 & 3 & 4 & 5 & 6 & Mean \\
\hline \multicolumn{16}{|c|}{2014} \\
\hline Salicylic acid & 11.25 & 11.68 & 12.10 & 12 & 13. & 14.10 & $\mid 12.49$ & 11.2. & 50 & $11.80 \mid 1$ & & & 2. & 7 & 12.09 \\
\hline Paraffin oil & 11.25 & 11.90 & 12.29 & 12.76 & 13.00 & 14.00 & 12.53 & 11.25 & 11.60 & 11.88 & 12.05 & 12.26 & 12.73 & 13.85 & 12.23 \\
\hline Camphor oil & 11.25 & 11.90 & 12.25 & 12.90 & 13.10 & 13.60 & 12.50 & 11.25 & 11.33 & $11.50 \mid 1$ & 11.60 & 11.80 & 12.18 & 13.10 & 11.82 \\
\hline Foil paper & 11.25 & 11.83 & 11.90 & 12.58 & 12.96 & 13.34 & 12.31 & 11.25 & 11.30 & 11.361 & 11.48 & 11.65 & 12.08 & 12.60 & 11.67 \\
\hline Control & 11.25 & 12.35 & 12.86 & 13.35 & & 15.10 & 13.14 & 11.2 & & $12.30 \mid 1$ & 12.68 & $12.8 \varepsilon$ & 13.18 & 14.17 & 12.63 \\
\hline Mean & 11.25 & 11.93 & 12.28 & 12.83 & 13. & 14.03 & & 11.25 & .53 & $11.77 \mid 1$ & 11.95 & 12.15 & 12.53 & 13.44 & \\
\hline LSD 5\% & A: & & B: 0.5 & & B: 1 & & & $\mathbf{A}:[$ & .35 & B: 0.3 & & & 0.91 & & \\
\hline \multicolumn{16}{|c|}{2015} \\
\hline Salicylic acid & 0.80 & 11.35 & $\mid 11.95$ & 12.75 & 13.32 & 14.41 & 12.43 & 10.80 & 11.05 & $11.46 \mid 1$ & 11.68 & 11.92 & 12.30 & 13.00 & 11.74 \\
\hline Paraffin oil & 10.80 & 11.20 & 12.35 & 12.80 & 13.15 & 14.22 & 12.42 & 10.80 & 11.11 & \begin{tabular}{l|l}
11.53 & 1 \\
\end{tabular} & 11.75 & 12.00 & 12.58 & 13.33 & 11.87 \\
\hline Camphor oil & 10.80 & 11.40 & 12.26 & 12.95 & 13.28 & 13.95 & 12.44 & 10.80 & 11.00 & \begin{tabular}{ll|l}
11.35 & 1
\end{tabular} & 11.48 & 11.85 & 12.15 & 12.66 & \begin{tabular}{|l|l|}
51.61 \\
\end{tabular} \\
\hline Foil paper & 10.80 & 11.30 & 11.93 & 12.65 & & 13.67 & 12.24 & 10.8 & 10.90 & 11.161 & 11.35 & & 12.10 & 12.10 & 11.49 \\
\hline Control & 10.80 & 11. & 12.15 & 13. & & 15.45 & 12.95 & 10.8 & & 12.101 & 43 & & 13.20 & 13.60 & 12.2 \\
\hline Mean & 10.80 & 11.42 & 12.13 & 12.89 & 13 & 14.34 & & 10.8 & 1.09 & \begin{tabular}{ll|l}
11.52 & 1 \\
\end{tabular} & 11.74 & & 12.4 & 12.99 & \\
\hline LSD 5\% & A: & & B: $: 0.4$ & & B: 1.0 & & & & .31 & B: $: 0.3$ & & $\mathbf{A B}$ & 0.86 & & \\
\hline
\end{tabular}

Table 5. Effect of coating and wrapping materials on titratable acidity of Balady mandarin fruits during storage in 2014 and 2015 seasons.

\begin{tabular}{|c|c|c|c|c|c|c|c|c|c|c|c|c|c|c|c|}
\hline \multirow{3}{*}{ Treat. (B) } & \multicolumn{7}{|c|}{ Room temperature } & \multicolumn{8}{|c|}{ Cold storages } \\
\hline & \multicolumn{7}{|c|}{ Weekly } & \multicolumn{8}{|c|}{ Biweekly } \\
\hline & P0 & 1 & 2 & 3 & 4 & 5 & Mean & P0 & 1 & 2 & 3 & 4 & 5 & 6 & Mean \\
\hline \multicolumn{16}{|c|}{2014} \\
\hline Salicylic acid & 1.32 & 1.20 & 1.03 & 0.95 & 0.78 & 0.75 & 1.01 & 1.32 & 1.26 & 1.23 & 1.09 & 0.99 & 0.90 & 0.83 & 1.08 \\
\hline Paraffin oil & 1.32 & 1.25 & 1.06 & 0.99 & 0.80 & 0.77 & 1.03 & 1.32 & 1.28 & 1.26 & 1.11 & 1.01 & 0.93 & 0.86 & 0.11 \\
\hline Camphor oil & 1.32 & 1.20 & 1.03 & 0.96 & 0.82 & 0.70 & 1.01 & 1.32 & 1.27 & 1.20 & 1.08 & 0.98 & 0.89 & 0.86 & 1.08 \\
\hline Foil paper & 1.32 & 1.22 & 1.06 & 1.01 & 0.84 & 0.71 & 1.03 & 1.32 & 1.25 & 1.18 & 1.03 & 1.09 & 0.84 & 0.89 & 1.08 \\
\hline Control & 1.32 & 1.18 & 1.01 & 0.87 & 0.71 & 0.64 & 0.96 & 1.32 & 1.21 & 1.11 & 1.00 & 0.92 & 0.80 & 0.76 & 1.02 \\
\hline Mean & 1.32 & 1.21 & 1.04 & 0.95 & 0.79 & 0.71 & & 1.32 & 1.25 & 1.19 & 1.06 & 0.99 & 0.87 & 0.84 & \\
\hline LSD 5\% & A: & 0.03 & \begin{tabular}{l|l} 
B: & 0.0 \\
\end{tabular} & \begin{tabular}{|l|l}
4 & $\mathrm{~A}$ \\
\end{tabular} & B: 0. & & & $\mathbf{A :}$ & 0.03 & \begin{tabular}{l|l} 
B: & 0. \\
\end{tabular} & & AB: & 0.09 & & \\
\hline \multicolumn{16}{|c|}{2015} \\
\hline Salicylic acid & 1.28 & 1.14 & 1.01 & 0.95 & 0.82 & 0.73 & 0.99 & 1.28 & 1.19 & 1.08 & 1.02 & 0.91 & 0.82 & 0.73 & 1.00 \\
\hline Paraffin oil & 1.28 & 1.18 & 1.08 & 1.01 & 0.86 & 0.73 & 1.02 & 1.28 & 1.20 & 1.10 & 1.00 & 0.93 & 0.86 & 0.78 & 1.02 \\
\hline Camphor oil & 1.28 & 1.15 & 1.01 & 0.98 & 0.80 & 0.67 & 0.98 & 1.28 & 1.18 & 1.10 & 1.01 & 0.94 & 0.85 & 0.79 & 1.02 \\
\hline Foil paper & 1.28 & 1.17 & 1.08 & 0.93 & 0.81 & 0.70 & 0.99 & 1.28 & 1.22 & 1.15 & 1.05 & 0.96 & 0.89 & 0.78 & 1.05 \\
\hline Control & 1.28 & 1.12 & 0.95 & 0.76 & 0.69 & 0.60 & 0.90 & 1.28 & 1.10 & 0.94 & 0.85 & 0.80 & 0.72 & 0.65 & 0.91 \\
\hline Mean & 1.28 & 1.15 & 1.03 & 0.93 & 0.79 & 0.69 & & 1.28 & 1.18 & 1.07 & 0.99 & 0.91 & 0.83 & 0.75 & \\
\hline LSD 5\% & A: & 0.03 & \begin{tabular}{l|l} 
B: & 0.0 \\
\end{tabular} & & B: 0. & & & A: & 0.02 & B: 10. & & AB: & 0.10 & & \\
\hline
\end{tabular}


Table 6. Effect of coating and wrapping materials on TSS/acid ratio of Balady mandarin fruits during storage in 2014 and 2015 seasons.

\begin{tabular}{|c|c|c|c|c|c|c|c|c|c|c|c|c|c|c|c|}
\hline \multirow{3}{*}{ Treat. (B) } & \multicolumn{7}{|c|}{ Room temperature } & \multicolumn{8}{|c|}{ Cold storages } \\
\hline & \multicolumn{7}{|c|}{ Weekly } & \multicolumn{8}{|c|}{ Biweekly } \\
\hline & P0 & 1 & 2 & 3 & 4 & 5 & Mean & P0 & 1 & 2 & 3 & 4 & 5 & 6 & Mean \\
\hline \multicolumn{16}{|c|}{2014} \\
\hline Salicylic acid & 8.52 & 9.73 & 11.75 & 13.34 & 16.86 & 18.70 & & 9.15 & 8.93 & 9.59 & 10.97 & 12.28 & 13.87 & 12.23 & \\
\hline Paraffin oil & .52 & 9.52 & & & 16.25 & 18.18 & & & 9.10 & .43 & & & 13.69 & & \\
\hline Camphor oil & 8.52 & 9.92 & 11.89 & 3.44 & 15.98 & 19.43 & & 9.15 & 6.89 & 9.58 & & 12.04 & 13.68 & 15.23 & 1.04 \\
\hline Foil paper & 8.52 & 9.67 & 11.23 & 4.98 & 16.62 & 18.79 & & 9.15 & 9.04 & 9.63 & 11 & 10.69 & 14.38 & 14.16 & 1.17 \\
\hline Control & 52 & 10.47 & 12.73 & 15.34 & 19.65 & 23.73 & 15.07 & 9.15 & 10.75 & 11.08 & 12 & & 16.47 & 18.64 & 3.25 \\
\hline Me & 8.52 & 9.86 & 11.84 & 13.98 & 17.07 & 18.17 & & 9.15 & 8.94 & 9.86 & 11.28 & 12.23 & 14.42 & 15.27 & 11.59 \\
\hline LSD 5\% & A: & 0.58 & B: & 0.44 & AB: & 1.12 & & A: & 0.83 & B: & 0.72 & AB: & 1.78 & & \\
\hline \multicolumn{16}{|c|}{2015} \\
\hline Salicy & 2.44 & 9.96 & 11.83 & 3.42 & 16.24 & 19.74 & & 8.44 & 9.29 & 10.61 & 11 . & 13.10 & 15.00 & 17.81 & 2.24 \\
\hline Paraff & 2.44 & 9.49 & 11.43 & 12.67 & 15.29 & 19.48 & & 8.44 & 9.26 & 10.48 & 11. & 12.90 & 14.63 & 17.09 & 2.08 \\
\hline Camphs & 2.44 & 9.91 & 12.14 & & 16.60 & 20.82 & & 4 & 9.3 & 10.32 & 11. & 61 & 14.29 & 16.02 & \\
\hline Foil paper & 2.44 & 9.66 & 11.05 & 13.60 & 16.18 & 19.53 & 13.08 & 8.44 & 8.93 & 9.70 & 10.81 & 12.22 & 13.59 & 15.90 & 11.37 \\
\hline Control & 2.44 & 12.47 & 12.79 & 17.54 & 20.43 & 25.75 & 16.24 & 8.44 & 10.39 & 12.87 & 14.62 & 15.71 & 18.33 & 20.92 & 14.47 \\
\hline Mean & 2.44 & 10.30 & 11.85 & 14.09 & 16.95 & 21.06 & & 8.44 & 9.44 & 10.79 & 12.00 & 13.31 & 15.17 & 17.55 & \\
\hline LSD 5\% & A: & 0.91 & B: & 0.85 & AB: & 2.06 & & A: & 0.86 & B: & 0.78 & AB: & 1.91 & & \\
\hline
\end{tabular}

Table 7. Effect of coating and wrapping materials on ascorbic acid of Balady mandarin fruits during storage in 2014 and 2015 seasons.

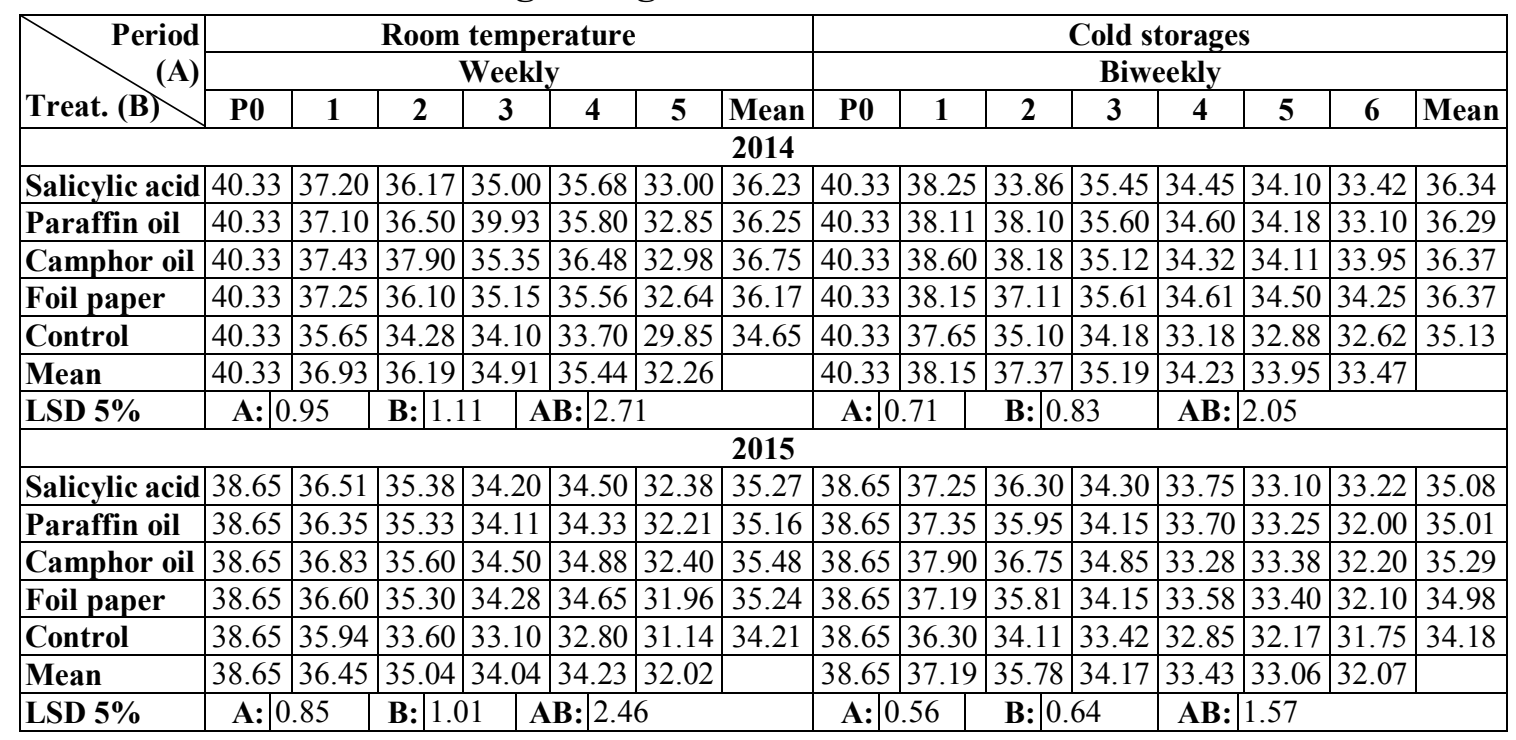


Table 8. Effect of coating and wrapping materials on total phenolics of Balady mandarin fruits during storage in 2014 and 2015 seasons.

\begin{tabular}{|c|c|c|c|c|c|c|c|c|c|c|c|c|c|c|c|}
\hline \multirow{3}{*}{ Period } & \multicolumn{7}{|c|}{ Room temperature } & \multicolumn{8}{|c|}{ Cold storages } \\
\hline & \multicolumn{7}{|c|}{ Weekly } & \multicolumn{8}{|c|}{ Biweekly } \\
\hline & P0 & 1 & 2 & 3 & 4 & 5 & Mean & P0 & 1 & 2 & \begin{tabular}{|l|l}
3 \\
\end{tabular} & 4 & 5 & 6 & Mean \\
\hline \multicolumn{16}{|c|}{2014} \\
\hline id & 35 & 1037 & 825 & 32 & 693 & \begin{tabular}{|l|l|}
661 & \\
\end{tabular} & 849 & 235 & & 70 & & & 951 & 27 & 07 \\
\hline $\mathbf{P}_{\mathbf{c}}$ & 35 & 10 & & & 676 & 638 & 56 & 35 & 165 & 171 & & & 943 & 30 & \\
\hline 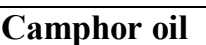 & 35 & 74 & & 7. & 699 & 655 & 8 & 235 & 318 & 1310 & & & 904 & & 23 \\
\hline ii & 1235 & 1041 & 895 & 748 & 701 & 688 & 885 & 1235 & 1365 & 1370 & 1186 & 1079 & 940 & 94 & 1153 \\
\hline & 1235 & 985 & 678 & & 493 & 463 & 730 & 1235 & 918 & 886 & 689 & 641 & 569 & 544 & 783 \\
\hline & 1235 & 1031 & 8 & 691 & 652 & 621 & & 1235 & 1188 & 1181 & 1038 & 965 & 841 & 836 & \\
\hline & A: 4 & 5.98 & B: 42 & \begin{tabular}{l|l}
39 & $A$ \\
\end{tabular} & B: 10 & 4.85 & & $\mathbf{A :}: 8$ & 5.56 & B: 80 & 0.78 & AB: & 191.4 & & \\
\hline \multicolumn{16}{|c|}{2015} \\
\hline & 290 & & & & 713 & 674 & 896 & 1290 & 1223 & 1228 & & $\mid 1064$ & 989 & 965 & 1127 \\
\hline & 1290 & 1052 & 55 & 743 & 705 & 663 & 886 & 1290 & 1220 & 1135 & 1062 & 980 & 980 & 958 & 1123 \\
\hline Camphor oil & 1290 & 1134 & 962 & 780 & 742 & 705 & 936 & 1290 & 1372 & 1296 & 1204 & 1108 & 938 & 910 & 1160 \\
\hline & 1290 & 1078 & 922 & 851 & 743 & 693 & 930 & 1290 & 1377 & 1409 & 1218 & 1133 & 963 & 945 & 1189 \\
\hline & 1290 & 1014 & & 55 & 523 & 491 & 753 & 1290 & 1063 & 991 & & \begin{tabular}{|l|}
654 \\
\end{tabular} & \begin{tabular}{|l|}
578 \\
\end{tabular} & & 842 \\
\hline & 1290 & 1072 & \begin{tabular}{|l|l}
867 \\
\end{tabular} & 736 & 685 & 645 & & 1290 & 1250 & 1229 & \begin{tabular}{|l|}
1054 \\
\end{tabular} & 1002 & 890 & 867 & \\
\hline & A: 6 & 5.92 & B: 55 & & B: 13 & 5.28 & & A: $: 7$ & 1.90 & B: 68 & 8.11 & AB: & 167.3 & & \\
\hline
\end{tabular}

In the contrary, under cold storage, acidity percentage, ascorbic acid (V.C) content and total phenolic content were higher values than under room temperature condition.

At the end of storage period, fruits of control samples had significantly the highest level of TSS (15.10 $\& 15.45)$ and (14.17 \& 13.60) and TSS/acid ratio $(23.73 \& 25.75 \%)$ and (18.64 \& 20.92\%) under room temperature and cold storage during the two studied seasons, respectively. Whereas, TSS values were (14.10, $14.0,13.60 \& 13.34)$ and (14.41, $14.22, \quad 13.95 \quad \& \quad 13.67 \%)$ and TSS/acid values were $(18.70,18.18$, $19.43 \& 18.79 \%)$ and $(19.74,19.48$, $20.82 \& 19.53)$ due to salicylic acid, paraffin oil, camphor oil and foil paper wrapping under room temperature during the two studied seasons, respectively. The corresponding values under cold storage were $13.47,13.85$, $13.10 \& 12.60$ and 13.00, 13.33, $12.66 \& 12.40 \%$ and $\mathrm{TSS} / \mathrm{acid}$ ratio were $12.23,16.10,15.23 \& 14.16$ and
$17.81,17.09,16.02 \& 15.90$, respectively.

On the other hand, at the end of storage period, untreated fruits had significantly the lowest values of acidity $(0.64 \& 0.60)$ and $(0.76 \&$ $0.65 \%)$, V.C. (29.85 \& 31.14) and (32.62 \& $31.75 \mathrm{mg} / 100 \mathrm{~g})$ and total phenolic (463 \& 491) and (544 \& 558 $\mathrm{mg} / 100 \mathrm{~g}$ ) under room temperature and cold storage during the two studied seasons, respectively. While, the highest values of these traits were observed due to oil coating or paper wrapping. The ascorbic acid values were $33.00,32.85,32.98 \& 32.64$ and $32.38,32.21,32.40 \& 31.96 \mathrm{mg} / 100 \mathrm{~g}$ as well as, total phenolic contents were 661, 638, $655 \& 686$ and 674, $663,705 \& 693 \mathrm{mg} / 100 \mathrm{~g}$ due to salicylic acid, paraffin oil, camphor oil and foil paper wrapping under room temperature during the two studied seasons, respectively. The corresponding values under cold storage were $33.42,33.10,33.95 \& 34.25$ and $32.22,32.00,32.20 \& 33.10 \mathrm{mg} / \mathrm{g}$, as 
well as total phenolic were 927, 930, $883 \& 894$ and $965,958,910 \& 945$ $\mathrm{mg} / 100 \mathrm{~g}$, respectively.

\section{Discussion}

Handling and storage is an important and of vital interest to fruit production and its quality. Fresh weight loss and fruit decay percentage were increased by extending storage duration. The loss of water from fruits during storage is a substantial problem due to shrinkage and weight loss, thus, the fruits could be damaged and loss its quality (BenYehoshua, 2005).

The loss of fruit weight indicated the tendency to lose water in fruits, which occurs during the fruits storage. The fruit weight decrease due to its respiratory process, the transference of humidity and some processes of oxidation and evaporation of moisture inside the fruits (Hassan et al., 2014).

Coating were used widely in fruits to reduce dehydration and water loss, prevents shriveling in fruit skin. Coating can act as a semi-permeable barrier against oxygen, carbon dioxide, moisture, so, they can reduce the rate of the respiration, water loss and oxidation reaction (Park, 1999). Salicylic acid control post-harvest losses in fruits through an inhibition of the ethylene biosynthesis and delay the senescence of different products by stimulating the accumulation of biologically active compounds and antioxidant enzymes (Gerailoo and Ghasemnezhad, 2011).

Decay of mandarin fruits during storage are mostly the cause of Penicillium sp. Moreover, the function of coating is a partial retention of gas exchange through the fruit peel and inhibition of the action of ethylene. Inhibition can give more prevention against post-harvest decay and water loss from the peel so decreasing the incidence of decay during storage (Abd El-Motty and El-Faham, 2013).

Coating and wrapping reduce respiration rates and may, therefore delay the utilization of organic acids (Cong et al., 2007).

The decreasing in decay percentage of treated fruits was probably due to the effects of coatings and wrapping on delaying senescence, which makes the commodity more vulnerable to pathogenic infection as a result of loss of cellular or tissue integrity (Patricia et al., 2005).

The results are in accordance with those obtained by Baldwin et al. (1995), Rodov et al. (2001), Plooy et al. (2009), Abd El-Motty and ElFaham (2013) and Mansour (2015).

It is known that during storage there is an antagonistic relation between TSS and acidity, while water loss and TSS were increased the acidity was decreased due to the process of respiration in fruits, because fruits consume acidity for respiration thereby total soluble solids increase more than acidity, thus TSS/acid ratio increases. The decrease in acid content is caused by the use of acids in the fruit as a source of energy and the conversion of organic acids to form sugar (Wills et al., 1998).

Antioxidant activity in fruits is high at the beginning of storage. This increase is due to resistance to chemical and enzymatic oxidation of polyphenols and antioxidants, thereby preventing loss of antioxidants during storage. Decreasing in antioxidants activity is associated with a decrease 
in the amount of phenolic compounds and ascorbic acid. The antioxidant capacity of citrus fruits was reduced during storage. Lower level of ascorbic acid in untreated fruits might be due to increased respiration. Ascorbic acid is su $0 \quad$ sceptible to oxidative deterioration or the formation of dehydroascorbic acid (Wills et al., 1998). Coating reduces oxygen levels and results in lower phenolic and antioxidant compounds during storage (Tietel et al., 2010).

The above mentioned findings are in accordance with those obtained by Saucedo et al. (2007), Plooy et al. (2009), Tietel et al. (2010), Abd ElMotty and El-Faham (2013) and Mansour (2015).

\section{Conclusion}

According to the previous results, it could be concluded that oil coating or wrapping maintained fruit freshness without negative effects on fruit quality parameters. Meanwhile, coating and wrapping fruits retained high levels of juice volume, acidity, ascorbic acid (V. C) and total phenolic contents as well as lower total soluble solids and TSS/acid ratio losses than controls during storage. Such treatments use instead chemicals are preferred in order to maintain human health and environment.

\section{References}

A.O.A.C. (1985): Official Method Analysis. Association of Official Analytical Chemists, Washington, DC., USA.

Abd El-Motty, Z. and S.Y. El-Faham (2013). Effect of Oil Coating and Different Wrapping Materials on Prolonging Storage Periods of Florida Prince Peach Fruits. Journal of Applied Sciences Research, 9(4): 2927-2937.
Aghdam, M.S. and S. Bodbodak (2013). Physiological and biochemical mechanisms regulating, chilling tolerance in fruits and vegetables under post-harvest salicylates and jasmonates treatments. Scientia Horticulture, 156: 73-85.

Baldwin, E.A.; M. Nisperos-Carriedo; P.E. Shaw and J.K. Burns (1995). Effect of coatings and prolonged storage conditions on fresh orange flavor volatiles, degrees, brix and ascorbic acid levels. Journal of Agricultural and Food Chemistry, 43 (5): 1321-1331.

Baubaker, H.; B. Saadi; E.H. Boudyach and Benaoumar (2009). Sensitivity of Penicillium digitatum and $P$. italicum to imazalil and Thiabendazole in Morocco. Plant Pathology Journal, 8 (4): 152-158.

Ben-Yehoshua, S. (2005). Individual, seal packaging of fruits and vegetables in plastic film-a new postharvest technique. HortScience 20: 32-37.

Cong, F.; Y. Zhang and W. Dong (2007). Use of surface coatings with natamycin to improve the storability of Hami melon at ambient temperature. Postharvest Biol. Technol., 46: 71-75.

Gerailoo, S. and M. Ghasemnezhad (2011). Effect of salicylic acid on antioxidant enzyme activity and petal senescence in yellow Island cut rose flowers. Journal of Fruit and Ornamental Plant Research, 19: 183-193.

Hassan, Z.H.; S. Lesmayati; R. Qomariah and A. Hasbianto (2014). Effect of wax coating applications and storage temperatures on the quality of tangerine citrus (Citrus reticulata) var. Siam Banjar. Int. Food Res. J., 21(2): 641-648.

Heber, D. and Q.Y. Lu (2002). Overview of mechanisms of action of lycopene. The Society for Experimen- 
tal Biology and Medicine, 227: 920-923.

Kader, A.A. (2002). Modified atmospheres during transport and storage. In. Post-Harvest technology of Horticultural Crops (Ed.). Univ. Calif. Div. Agr. Nat. Res. Publ. 3311 Oakland, CA pp. 135-144.

Ladaniya, M.S. (2011). Physicochemical, respiratory and fungicide residue changes in wax coated mandarin fruit stored at chilling temperature with intermittend warming. J. Food Sci. Technol., 48 (2): $150-158$.

Mansour, A.H. (2015). Effect of pre and post-harvest treatments on fruit characteristics of Balady mandarin (Citrus reticulata L.) during storage. Ph.D. Thesis, Fac. of Agric., Assiut Univ., Egypt.

Park, H.J. (1999). Development of advanced edible coatings for fruits. Trends Food Sci. Technol., 10: 254-260.

Patricia, S.; T. Palmu and C.R.F. Grosso (2005). Effect of edible wheat gluten- based films and coating on refrigerated strawberry (Fragaria ananassa) quality. Postharvest Biol.Technol., 36: 199-208.

Plooy, W.; T. Regnier and S. Combrinck (2009). Essential oil amended coatings as alternatives to synthetic fungicides in citrus post-harvest management. Post-harvest Biology and Technology, 53: 117-122.

Rodov, V.; T. Agar; J. Peretz; B. Nafussi; Kim Jong, and S. BenYehoshua (2001). Effect of com- bined application of heat treatments and plastic packaging on keeping quality of "oroblanco" fruit (Citrus grandis L. Xc. Paradisimacr). Post-harvest Biology and Technology, 20 (3): 287-294. (c.f. Hort. Abst. 71 (4), 2911).

Saucedo, P.S.; D. Jasso-Cantu; J. Ventura-Sabrevilla; A. Saenz-Galindo; R. Rodriguez-Herrera and C.N. Aguilar (2007). Effect of canenlla wax with natural antioxidants on the shelf life quality of fresh cut fruits. J. of Food Quality, 30 (5): 823-836.

Singleton, V.L.; R. Orthofer and R.S. Lamuela (1999). Analysis of total phenols and other oxidation substrates and antioxidants by means of Folin-Ciacalteau Reagent. Methods Enzymol, 299: 152-178.

Snedecor, G.W. and W.G. Cochran (1990): Statistical Methods $7^{\text {th }}$ Edn, Iowa State University Press, Ames, IA, USA.

Tietel, Z.; E. Lewinsohn; E. Feldmesser; F. Fallik and R. Porat (2010). Effect of wax coatings and postharvest storage on sensory quality and aroma volatile composition of "Mor" mandarins. J. of Sci. of Food Agric., 90 (6): 995-1007.

Wills, R.B.H.; W.B. Me Glasson; D. Graham and D. Joyce (1998). Postharvest Introduction to the physiology and handling of fruits, vegetable and ornamentals. $4^{\text {th }} \mathrm{New}$ South Wales University Press Limited, Kensington, England. 
تأثير مواد التغطية واللف علي السلوك التخزيني لثمار اليوسفي البلاي

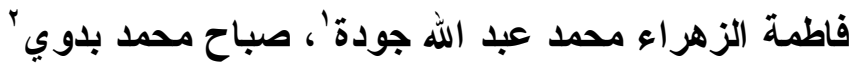

$$
\begin{aligned}
& \text { ' 'قسم الفاكهة - كلية الزراعة- جامعة أسيوط } \\
& \text { r مركز البحوث الزراعية - شندويل - سوهاج }
\end{aligned}
$$

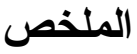

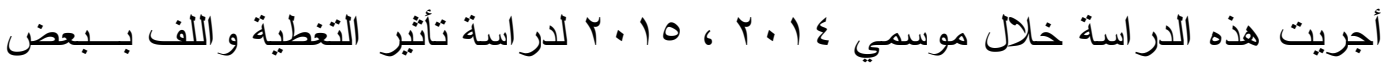

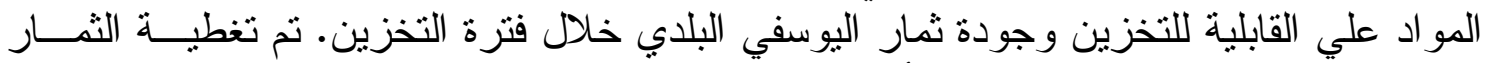

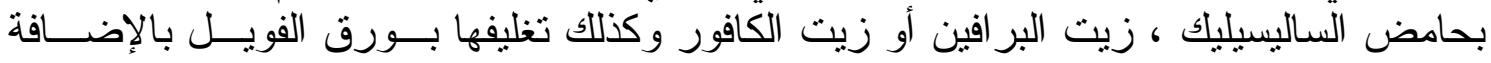

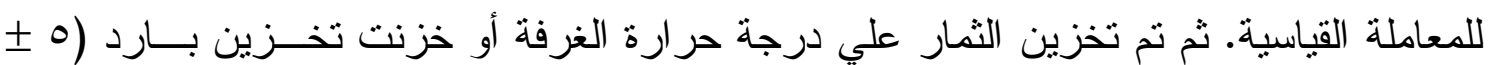

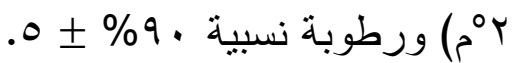

أوضحت النتائج أن النسبة المئوية للفقد في الوزن و التالف و المو اد الصلبة الذائبـــة الكليـــة

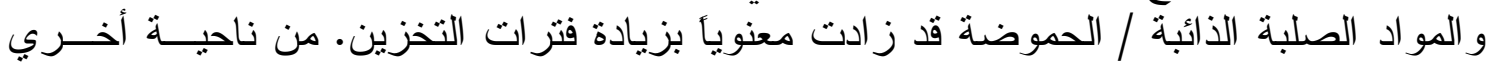

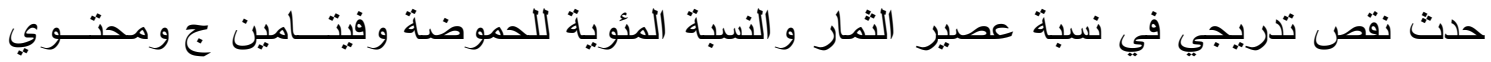
الفينو لات الكلية بزيَّادة مدة في التخزين.

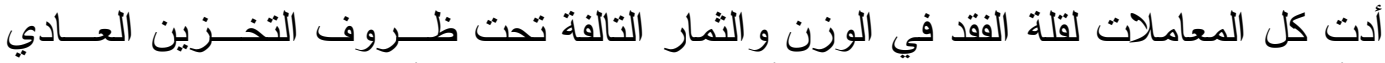

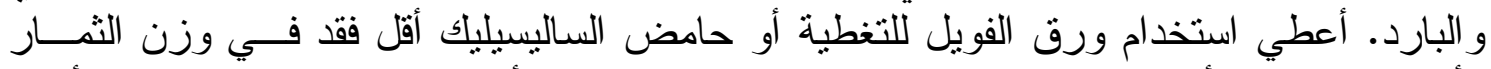

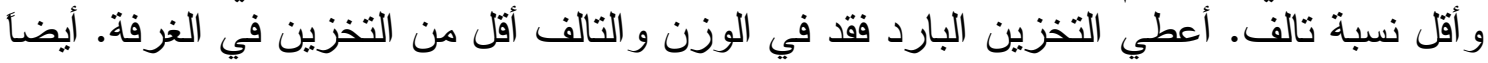
حسنت كل المعاملات جودة الثمار خلال التل التخزين مقارنة بالكنترول.

يمكن القول بأن التغطية بالزيوت أو لف الثمار بحافظ علي الثمار طازجة بدون تـأثنير ات

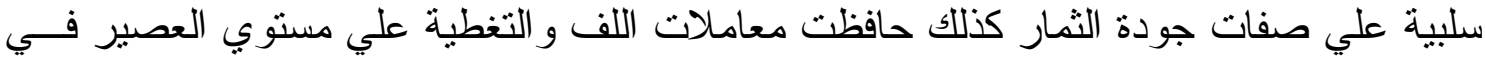

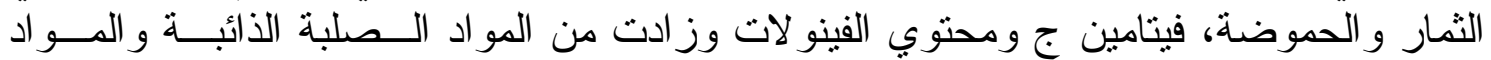
الصلبة الذائبة / الحموضة. لذا يمكن استخدام هذه المعاملات بديلا للمو اد الكينة لإن الكياوية وذلك للحفاظ علي الصحة العامة و البيئة. 